Pacific

Journal of

Mathematics

\title{
COTILTING MODULES AND BIMODULES
}

Riccardo Colpi and Kent R. Fuller 


\title{
COTILTING MODULES AND BIMODULES
}

\author{
Riccardo Colpi and Kent R. Fuller
}

\begin{abstract}
Cotilting modules and bimodules over arbitrary associative rings are studied. On the one hand we find a connection between reflexive modules with respect to a cotilting (bi)module $U$ and a notion of $U$-torsionless linear compactness. On the other hand we provide concrete examples of cotilting bimodules over linearly compact noetherian serial rings.
\end{abstract}

Cotilting theory is a generalization of Morita duality in a sense that is analogous to that in which tilting theory is a generalization of Morita equivalence. Indeed, cotilting modules first appeared as vector space duals of tilting modules over finite dimensional algebras (see, e.g., [H, IV, 7.8]), just as injective cogenerators are such duals of progenerators. Later, R.R. Colby [Cb1] studied finitely generated cotilting bimodules over noetherian rings, proving that they induce finitistic generalized Morita dualities, similar to the finite dimensional algebra case. More recently, in [Cb2] he investigated a more general class of representable dualities, namely (nonfinitistic) generalized Morita dualities. He proved that the existence of such a duality implies the existence of a second pair of functors between classes that complement the reflexive ones, obtaining a result which is close to a dual form of the celebrated Tilting Theorem $[\mathbf{B r B u}],[\mathbf{H a R i}]$.

For arbitrary rings $R$ and $S$, a Morita duality between left $S$-modules and right $R$-modules is given by the contravariant Hom functors induced by a so called Morita bimodule ${ }_{S} W_{R}$, namely, one such that (i) the classes of $W$-reflexive modules contain $R_{R}, W_{R},{ }_{S} S$ and ${ }_{S} W$, and are closed under submodules, factor modules and extensions; or, equivalently, (ii) ${ }_{S} W_{R}$ is balanced, and $W_{R}$ and ${ }_{S} W$ are injective cogenerators. Colby's generalized Morita dualities in [Cb2] are those induced by a bimodule ${ }_{S} U_{R}$ such that a natural weakening of (i) holds (just closure under factor modules is left out). Generalizing the notion of injective cogenerator, the authors of [CpDeTo] and $[\mathbf{C p} \mathbf{T o} \mathbf{T r}]$ defined a cotilting module $U_{R}$ over a ring $R$ as one such that $\operatorname{Cogen}\left(U_{R}\right)=\operatorname{Ker} \operatorname{Ext}_{R}^{1}\left(-, U_{R}\right)$. In [CpDeTo, Proposition 1.7] it is shown that this notion is dual to that of tilting module by means of the following

Proposition. A module $U_{R}$ is a cotilting module if and only if it satisfies the conditions

(1) $\operatorname{inj} \operatorname{dim}\left(U_{R}\right) \leq 1$, 
(2) $\operatorname{Ext}_{R}^{1}\left(U_{R}^{\alpha}, U_{R}\right)=0$ for any cardinal $\alpha$,

(3) $\operatorname{Ker}_{H^{\prime}}\left(-, U_{R}\right) \cap \operatorname{Ker} \operatorname{Ext}_{R}^{1}\left(-, U_{R}\right)=0$.

To obtain a homological generalization of (ii), as in $[\mathbf{C p}]$, we say that a balanced bimodule ${ }_{S} U_{R}$ in which both $U_{R}$ and ${ }_{S} U$ are cotilting modules is a cotilting bimodule.

In this paper we continue the study of cotilting (bi)modules over arbitrary rings that was begun in $[\mathbf{C p}]$. There it was shown that any cotilting bimodule ${ }_{S} U_{R}$ induces a pair of dualities between quite large subcategories of torsion-free and torsion modules in Mod- $R$ and $S$-Mod, respectively. This result naturally generalizes Morita dualities to torsion theories, and it is still dual to the Tilting Theorem.

A third major component of Morita duality theory is B. Müller's theorem [X, Corollary 4.2] that the reflexive modules relative to a Morita bimodule are precisely the linearly compact modules. In Section 1 we investigate the related notion of torsionless linear compactness and its connection to the reflexivity of modules. This allows us to find a bridge between Colby's generalized Morita duality and cotilting bimodules by showing that a cotilting bimodule $U$ induces a generalized Morita duality if and only if the classes of the $U$-reflexive modules coincide with those of the $U$-torsionless linearly compact modules. This is accomplished, in part, by answering a question posed in $[\mathbf{C p}]$.

Perhaps the most accessible collection of examples of tilting modules over non-artinian rings are those over hereditary noetherian serial rings. They and their endomorphism rings were classified in $[\mathbf{C b F u}]$. In Section 2 we show that the Morita dual of a tilting module possesses most of the properties of a cotilting bimodule. Then in Section 3 we employ these results and Warfield's theorems on noetherian serial rings in [Wa] to show that the dual of any tilting module over a noetherian serial ring with selfduality is a cotilting bimodule. Thus we obtain a class of concrete examples of cotilting bimodules that are not, in general, finitely generated.

We denote by $R$ and $S$ two arbitrary associative rings with unit, and by Mod- $R$ and $S$-Mod the category of all unitary right $R$ - and left $S$-modules, respectively. All the classes of modules that we introduce are to be considered as full subcategories of modules closed under isomorphisms. Given a module $U$, we denote by $\operatorname{add}(U)$ the class of all direct summands of any finite direct sum of copies of $U$, and by $\operatorname{Cogen}(U)$ the class of all modules cogenerated by $U$, that is all the modules $M$ such that there exists an exact sequence $0 \rightarrow M \rightarrow U^{\alpha}$, for some cardinal $\alpha$. We denote by $\operatorname{Rej}_{U}(-)$ the reject radical, defined by $\operatorname{Rej}_{U}(M)=\cap\left\{\operatorname{Ker}(f) \mid f \in \operatorname{Hom}_{R}(M, U)\right\}$, i.e., the least submodule $M_{0}$ of $M$ such that $M / M_{0}$ belongs to $\operatorname{Cogen}(U)$. Given a bimodule ${ }_{S} U_{R}$, we denote by $\Delta$ both the functors $\operatorname{Hom}_{R}(-, U)$ and $\operatorname{Hom}_{S}(-, U)$, and by $\Gamma$ both the functors $\operatorname{Ext}_{R}^{1}(-, U)$ and $\operatorname{Ext}_{S}^{1}(-, U)$. For 
any module $M, \delta_{M} M \rightarrow \Delta^{2}(M)$ denotes the evaluation morphism. $M$ is called $\Delta$-reflexive if $\delta_{M}$ is an isomorphism. Note that if $U_{R}$ is a cotilting module, then $(\operatorname{Ker} \Delta, \operatorname{Ker} \Gamma)$ is a torsion theory in $\operatorname{Mod}-R$, associated to the idempotent radical $\operatorname{Rej}_{U}(-)=\operatorname{Ker}\left(\delta_{-}\right)$. For further notation, we refer to $[\mathbf{A F}],[\mathbf{S}]$ and $[\mathbf{C E}]$.

\section{Reflexivity and torsionless linear compactness.}

We start this section pointing out some facts on $\Delta$-reflexivity of modules, with respect to a cotilting module $U_{R}$, which generalize part of $[\mathbf{C p}$, Lemma 4 and Proposition 5]:

Lemma 1.1. Let $U_{R}$ be a cotilting module, and let $S=\operatorname{End}\left(U_{R}\right)$. Then:

(a) $U_{R}$ and ${ }_{S} S$ are $\Delta$-reflexive.

(b) If ${ }_{S} L \in S$-Mod is a factor of any $\Delta$-reflexive module (in particular, if ${ }_{S} L$ is finitely generated), then $\delta_{L}$ is an epimorphism.

(c) If ${ }_{S} L \in \operatorname{Cogen}\left({ }_{S} U\right)$ is a factor of any $\Delta$-reflexive module, then $L$ is $\Delta$-reflexive.

(d) For any $M_{R} \in \operatorname{Mod}-R$, we have $\operatorname{Coker}\left(\delta_{M}\right) \in \operatorname{Ker} \Gamma$.

(e) Let $M_{R} \in \operatorname{Mod}-R$. Then $M_{R}$ is $\Delta$-reflexive if and only if $M_{R} \in \operatorname{Ker} \Gamma$ and $\Delta(M)$ is $\Delta$-reflexive.

(f) If $M_{R} \in \operatorname{Ker} \Gamma$ and $\Delta\left(M_{R}\right)$ is a factor of any $\Delta$-reflexive module (in particular, if $\Delta\left(M_{R}\right)$ is finitely generated), then $M_{R}$ is $\Delta$-reflexive.

(g) If $L_{R} \leq M_{R}, M_{R}$ is $\Delta$-reflexive and $M / L \in \operatorname{Ker} \Gamma$, then $L_{R}$ is $\Delta$ reflexive.

Proof. (a) $\Delta^{2}\left(U_{R}\right) \cong \Delta\left({ }_{S} S\right) \cong U_{R}$ and $\Delta^{2}\left({ }_{S} S\right) \cong \Delta\left(U_{R}\right) \cong{ }_{S} S$ canonically.

(b) Let $K \rightarrow M \rightarrow L \rightarrow 0$ be an exact sequence in $S$-Mod, with $M$ $\Delta$-reflexive. Then we have the exact sequence $0 \rightarrow \Delta(L) \rightarrow \Delta(M) \rightarrow I \rightarrow$ 0 , where $I$ embeds into $\Delta(K)$, so that $\Gamma(I)=0$. Therefore we get the commutative exact diagram

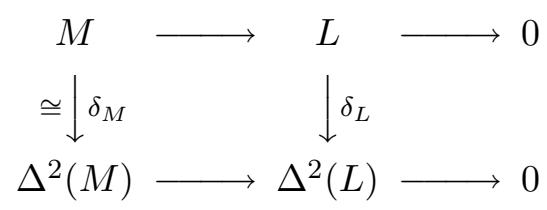

which shows that $\delta_{L}$ is epic.

(c) Clearly ${ }_{S} L \in \operatorname{Cogen}\left({ }_{S} U\right)$ if and only if $\delta_{L}$ is monic. We can conclude by (b).

(d) By adjunction, we get $\Delta\left(\delta_{M}\right) \circ \delta_{\Delta(M)}=\mathrm{id}_{\Delta(M)}$, so that $\Delta\left(\delta_{M}\right)$ is epic. Therefore, from the exact sequence $0 \rightarrow M / \operatorname{Rej}_{U}(M) \rightarrow \Delta^{2}(M) \rightarrow \operatorname{Coker}\left(\delta_{M}\right)$ $\rightarrow 0$ we see that $\Gamma\left(\operatorname{Coker}\left(\delta_{M}\right)\right) \hookrightarrow \Gamma \Delta^{2}(M)=0$.

(e) Again from the identity $\Delta\left(\delta_{M}\right) \circ \delta_{\Delta(M)}=\operatorname{id}_{\Delta(M)}$, we see that if $\delta_{M}$ is an isomorphism, then $\delta_{\Delta(M)}$ is too, and of course $M \in \operatorname{Cogen}\left(U_{R}\right)=\operatorname{Ker} \Gamma$. 
Conversely, if $\delta_{\Delta(M)}$ is an isomorphism, then $\Delta\left(\delta_{M}\right)$ must be monic, i.e., $\operatorname{Coker}\left(\delta_{M}\right) \in \operatorname{Ker} \Delta$. Moreover $\operatorname{Coker}\left(\delta_{M}\right) \in \operatorname{Ker} \Gamma$ because of $(\mathrm{d})$. Since $(\operatorname{Ker} \Delta, \operatorname{Ker} \Gamma)$ is a torsion theory, we conclude that $\operatorname{Coker}\left(\delta_{M}\right)=0$, i.e., $\delta_{M}$ is epic. Under the further assumption that $M \in \operatorname{Ker} \Gamma$, we conclude that $\delta_{M}$ is an isomorphism.

(f) Since $\Delta(M) \in \operatorname{Cogen}\left({ }_{S} U\right)$, (c) applies, giving $\Delta(M)$ reflexive. We conclude by (e).

(g) From the exact sequence $0 \rightarrow L \rightarrow M \rightarrow M / L \rightarrow 0$ in Mod- $R$, by assumption we get the exact sequence $0 \rightarrow \Delta(M / L) \rightarrow \Delta(M) \rightarrow \Delta(L) \rightarrow 0$. We conclude using (e) and (f).

It is well known that linear compactness plays a fundamental role in the study of duality. Here we introduce a concept of linear compactness with respect to a torsion theory, drawing inspiration from [GpGaWi, §3]:

Definition 1.2. Let $(\mathcal{T}, \mathcal{F})$ be a torsion theory in Mod- $R$. Then a right $R$-module $M$ is called $\mathcal{F}$-linearly compact if $M \in \mathcal{F}$ and for any inverse system of morphisms $\left\{p_{\lambda}: M \rightarrow M_{\lambda}\right\}$ with $M_{\lambda} \in \mathcal{F}$ and $\operatorname{Coker}\left(p_{\lambda}\right) \in \mathcal{T}$, for all $\lambda$ 's, it happens that Coker $\left(\lim _{p_{\lambda}}\right) \in \mathcal{T}$.

If $U_{R}$ is a cotilting module, a module $M \in \operatorname{Mod}-R$ is called $U$-torsionless linearly compact ( $U$-tl.l.c., for short) if $M$ is $\operatorname{Ker} \Gamma$-linearly compact.

Note that $M \in \operatorname{Mod}-R$ is linearly compact iff $M$ is Mod- $R$-linearly compact, i.e., it is linearly compact with respect to the trivial torsion theory $(\{0\}, \operatorname{Mod}-R)$. In particular if $U_{R}$ is a cotilting module, then the $U$ torsionless linear compactness coincides with the usual linear compactness iff $U_{R}$ is an injective cogenerator.

Torsionfree linear compactness is inherited by any inverse limit of the type in Definition 1.2, as the following result due to A. Tonolo shows:

Proposition 1.3. Let $(\mathcal{T}, \mathcal{F})$ be a torsion theory in Mod-R, and let $M \in$ Mod- $R$ be $\mathcal{F}$-linearly compact. Then for any inverse system $\left\{p_{\lambda}: M \rightarrow M_{\lambda}\right\}$ with $M_{\lambda} \in \mathcal{F}$ and $\operatorname{Coker}\left(p_{\lambda}\right) \in \mathcal{T}$, the module $\varliminf_{\lambda} M_{\lambda}$ is $\mathcal{F}$-linearly compact too.

Proof. First of all, let us note that $\lim _{M_{\lambda}} M_{\lambda} \in \mathcal{F}$, because $\mathcal{F}$ is closed under inverse limits. Next, let $\left\{p_{\mu}^{\prime}: \underset{\lim }{\longleftarrow} M_{\lambda} \rightarrow M_{\mu}^{\prime}: \mu \in \Lambda^{\prime}\right\}$ be any inverse system with $M_{\mu}^{\prime} \in \mathcal{F}$ and $\operatorname{Coker}\left(p_{\mu}^{\prime}\right) \in \mathcal{T}$ for all $\mu$ 's. Let us prove that Coker $\left(\lim _{\longleftarrow} p_{\mu}^{\prime}\right) \in \mathcal{T}$. Note that the cokernel of each map $p_{\mu}^{\prime} \circ \lim _{\longleftarrow} p_{\lambda}$, for all $\mu$ 's, is in $\mathcal{T}$, because it is an extension of a factor of Coker $\left(\lim _{p_{\lambda}}\right)$, which is in $\mathcal{T}$, by the torsion module $\operatorname{Coker}\left(p_{\mu}^{\prime}\right)$. Hence, by assumption, we get that

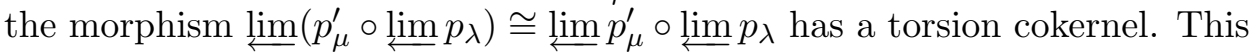

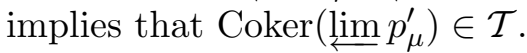

In $\left[\mathbf{C p}\right.$, Proposition 10] it was proved that if ${ }_{S} U_{R}$ is a cotilting bimodule, then any $U$-tl.l.c. module is $\Delta$-reflexive; and the question of whether the 
converse is true was posed. To give a partial answer, we start with a theorem which generalizes a well known result, substantially due to Müller [Mu] (see also [X, Theorem 4.1]):

Theorem 1.4. Let $U_{R}$ be a cotilting module, and let $S=\operatorname{End}\left(U_{R}\right)$. Then the following are equivalent for any $M \in \operatorname{Mod}-R$ :

(1) $M$ is $U$-tl.l.c.

(2) $M$ is $\Delta$-reflexive, and for all $L \stackrel{i}{\hookrightarrow} \Delta(M)$ we have Coker $\Delta(i) \in \operatorname{Ker} \Delta$.

Proof. $(1) \Rightarrow(2)$. Let $M_{R}$ be $U$-tl.l.c., let $L$ be a submodule of $\Delta(M)$ and let $\left\{L_{\lambda}: \lambda \in \Lambda\right\}$ be the upward directed family of the finitely generated submodules of $L$. Thus, if we denote by $i_{\lambda}: L_{\lambda} \hookrightarrow L \stackrel{i}{\hookrightarrow} \Delta(M)$ the canonical inclusions, we get $\lim _{\longrightarrow} L_{\lambda}=L$ and $\lim _{\longrightarrow} i_{\lambda}=i$. Let now $p_{\lambda}=\Delta\left(i_{\lambda}\right) \circ \delta_{M}$ : $M \rightarrow \Delta\left(L_{\lambda}\right)$. Then $\left\{p_{\lambda}: \lambda \in \Lambda\right\}$ is an inverse system of morphisms in $\operatorname{Ker} \Gamma$. In order to show that $\operatorname{Coker}\left(p_{\lambda}\right) \in \operatorname{Ker} \Delta$ for any $\lambda$, let us consider the commutative diagram in $\operatorname{Mod}-R$

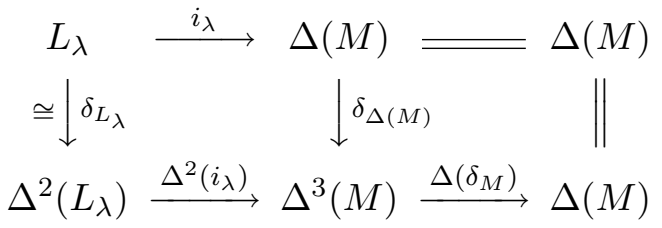

where $\delta_{L_{\lambda}}$ is an isomorphism because of Lemma 1.1(c), which proves that $\Delta\left(p_{\lambda}\right)=\Delta\left(\delta_{M}\right) \circ \Delta^{2}\left(i_{\lambda}\right)$ is monic, i.e., $\operatorname{Coker}\left(p_{\lambda}\right) \in \operatorname{Ker} \Delta$.

Thus the hypothesis (1) applies, giving $\operatorname{Coker}\left(\varliminf^{\lim } p_{\lambda}\right) \in \operatorname{Ker} \Delta$. Moreover

$$
\lim _{\longleftarrow} p_{\lambda}=\lim _{\longleftarrow} \Delta\left(i_{\lambda}\right) \circ \delta_{M} \cong \Delta\left(\lim _{\longrightarrow} i_{\lambda}\right) \circ \delta_{M}=\Delta(i) \circ \delta_{M} .
$$

First, if we choose $L=\Delta(M)$ we clearly get $\operatorname{Coker}\left(\delta_{M}\right) \cong \operatorname{Coker}\left(\lim p_{\lambda}\right) \in$ $\operatorname{Ker} \Delta$. On the other hand, since $M \in \operatorname{Ker} \Gamma, \delta_{M}$ is injective and $\operatorname{Coker}\left(\delta_{M}\right) \in$ Ker $\Gamma$ because of Lemma 1.1(d). Therefore $\operatorname{Coker}\left(\delta_{M}\right)=0$, i.e., $M$ is $\Delta$ reflexive.

Finally, in the case $L$ is arbitrary, since $\delta_{M}$ is an isomorphism, from $(*)$ we get $\operatorname{Coker}(\Delta(i)) \cong \operatorname{Coker}\left(\lim _{p_{\lambda}}\right) \in \operatorname{Ker} \Delta$.

$(2) \Rightarrow(1)$. Let $\left\{p_{\lambda}: M \rightarrow M_{\lambda}\right\}$ be an inverse system of morphisms in $\operatorname{Mod}-R$, with $M, M_{\lambda} \in \operatorname{Ker} \Gamma$ and $\operatorname{Coker}\left(p_{\lambda}\right) \in \operatorname{Ker} \Delta$ for all $\lambda$ 's.

In the sequel, we will refer to the following exact sequences

$$
0 \longrightarrow K_{\lambda} \longrightarrow M \stackrel{\alpha_{\lambda}}{\longrightarrow} I_{\lambda} \longrightarrow 0
$$

$$
0 \longrightarrow I_{\lambda} \stackrel{\beta_{\lambda}}{\longrightarrow} M_{\lambda} \longrightarrow C_{\lambda} \longrightarrow 0
$$

with $K_{\lambda}=\operatorname{Ker}\left(p_{\lambda}\right), I_{\lambda}=\operatorname{Im}\left(p_{\lambda}\right), C_{\lambda}=\operatorname{Coker}\left(p_{\lambda}\right)$ and $\beta_{\lambda} \circ \alpha_{\lambda}=p_{\lambda}$.

First, let us prove that all the $K_{\lambda}, I_{\lambda}, M_{\lambda}$ are $\Delta$-reflexive. Note that the sequence (ex1) is in $\operatorname{Ker} \Gamma$, and $M$ is $\Delta$-reflexive by assumption, so that from Lemma 1.1(g) we obtain that $K_{\lambda}$ is $\Delta$-reflexive too. Moreover, looking 
at the embedding $\Delta\left(\alpha_{\lambda}\right): \Delta\left(I_{\lambda}\right) \hookrightarrow \Delta(M)$, by hypothesis we have that $\operatorname{Coker}\left(\Delta^{2}\left(\alpha_{\lambda}\right)\right) \in \operatorname{Ker} \Delta$. Thus we obtain the commutative exact diagram 0

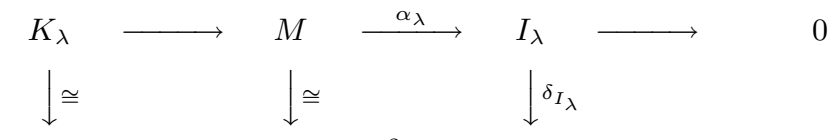

$0 \longrightarrow \Delta^{2}\left(K_{\lambda}\right) \longrightarrow \Delta^{2}(M) \stackrel{\Delta^{2}\left(\alpha_{\lambda}\right)}{\longrightarrow} \Delta^{2}\left(I_{\lambda}\right) \longrightarrow \operatorname{Coker}\left(\Delta^{2}\left(\alpha_{\lambda}\right)\right) \longrightarrow 0$

from which we get (thanks to Lemma 1.1(d)) $\operatorname{Coker}\left(\Delta^{2}\left(\alpha_{\lambda}\right)\right) \cong \operatorname{Coker}\left(\delta_{I_{\lambda}}\right) \in$ $\operatorname{Ker} \Gamma$. Thus $\operatorname{Coker}\left(\delta_{I_{\lambda}}\right)=0$, i.e., $I_{\lambda}$ is $\Delta$-reflexive. Next, from (ex2) we get the commutative exact diagram

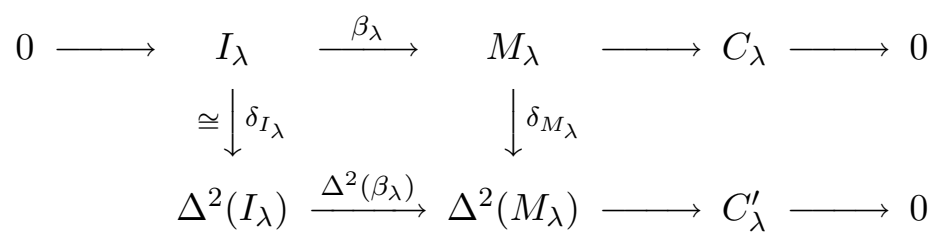

where $C_{\lambda} \in \operatorname{Ker} \Delta$ by assumption. Let us prove that $C_{\lambda}^{\prime} \in \operatorname{Ker} \Delta$ too. From the embedding

$$
0=\Delta\left(C_{\lambda}\right) \longrightarrow \Delta\left(M_{\lambda}\right) \stackrel{\Delta\left(p_{\lambda}\right)}{\longrightarrow} \Delta(M)
$$

we get, by hypothesis, that $\operatorname{Coker}\left(\Delta^{2}\left(p_{\lambda}\right)\right) \in \operatorname{Ker} \Delta$. From $\Delta^{2}\left(p_{\lambda}\right)=$ $\Delta^{2}\left(\beta_{\lambda}\right) \circ \Delta^{2}\left(\alpha_{\lambda}\right)$ we see that $C_{\lambda}^{\prime}=\operatorname{Coker}\left(\Delta^{2}\left(\beta_{\lambda}\right)\right) \in \operatorname{Ker} \Delta$ too. Therefore, applying the functor $\Delta$ to the previous diagram we obtain the commutative exact diagram

$$
\begin{gathered}
0 \longrightarrow \Delta\left(M_{\lambda}\right) \stackrel{\Delta\left(\beta_{\lambda}\right)}{\longrightarrow} \Delta\left(I_{\lambda}\right) \\
\downarrow \Delta\left(\delta_{M_{\lambda}}\right)
\end{gathered}
$$

which shows that $\Delta\left(\delta_{M_{\lambda}}\right)$ is monic. Since $\Delta\left(\delta_{M_{\lambda}}\right) \circ \delta_{\Delta\left(M_{\lambda}\right)}=\operatorname{id}_{\Delta\left(M_{\lambda}\right)}$, we conclude that $\delta_{\Delta\left(M_{\lambda}\right)}$ is an isomorphism, so that $M_{\lambda}$ is $\Delta$-reflexive, because of Lemma 1.1(e).

Finally, from (ex3), we derive the embedding $\lim _{\longrightarrow} \Delta\left(p_{\lambda}\right): \underline{\lim } \Delta\left(M_{\lambda}\right) \hookrightarrow$ $\Delta(M)$, so that $\operatorname{Coker}\left(\Delta\left(\lim _{\longrightarrow} \Delta\left(p_{\lambda}\right)\right)\right) \in \operatorname{Ker} \Delta$ by assumption. Therefore we get the commutative exact diagram

$$
\begin{aligned}
& \Delta^{2}(M) \stackrel{\Delta\left(\lim _{\longrightarrow} \Delta\left(p_{\lambda}\right)\right)}{\longrightarrow} \Delta\left(\stackrel{\lim }{\longrightarrow} \Delta\left(M_{\lambda}\right)\right) \cong \lim _{\longleftarrow} \Delta^{2}\left(M_{\lambda}\right) \longrightarrow \operatorname{Coker}\left(\Delta\left(\underline{\lim } \Delta\left(p_{\lambda}\right)\right)\right) \longrightarrow 0 \\
& \cong \uparrow \delta_{M} \\
& M \quad \stackrel{\lim p_{\lambda}}{\longrightarrow} \quad \stackrel{\lim }{\leftrightarrows} M_{\lambda} \quad \longrightarrow \quad \operatorname{Coker}\left(\stackrel{\lim }{\longleftarrow} p_{\lambda}\right)
\end{aligned}
$$

which shows that $\operatorname{Coker}\left(\varliminf_{\lim } p_{\lambda}\right) \cong \operatorname{Coker}\left(\Delta\left(\lim _{\longrightarrow} \Delta\left(p_{\lambda}\right)\right)\right) \in \operatorname{Ker} \Delta$.

The next result points out some good properties of $U$-tl.l.c. modules.

Corollary 1.5. Let $U_{R}$ be a cotilting module. 
(a) If $M \rightarrow M^{\prime} \rightarrow T \rightarrow 0$ is exact in Mod-R, and $M$ is $U$-tl.l.c., $M^{\prime} \in$ $\operatorname{Ker} \Gamma$ and $T \in \operatorname{Ker} \Delta$, then $M^{\prime}$ is $U$-tl.l.c. too.

(b) If $M \in \operatorname{Mod}-R$ is a factor of any $U$-tl.l.c. module, then $\delta_{M}$ is surjective and $M / \operatorname{Rej}_{U}(M)$ is $U$-tl.l.c. too.

Proof. (a) is an immediate consequence of Proposition 1.3. In order to prove (b), let us consider an epimorphism $L \stackrel{\varphi}{\rightarrow} M \rightarrow 0$, with $L U$-tl.l.c. From Theorem 1.4 we get that $L$ is $\Delta$-reflexive and, considering the embedding $0 \longrightarrow \Delta(M) \stackrel{\Delta(\varphi)}{\longrightarrow} \Delta(L)$, also that $\operatorname{Coker}\left(\Delta^{2}(\varphi)\right) \in \operatorname{Ker} \Delta$. On the other hand, from the commutative exact diagram

$$
\begin{array}{cccc}
L & \stackrel{\varphi}{\longrightarrow} \quad M \quad \longrightarrow \quad 0 \\
\cong \downarrow \delta_{L} & \downarrow \delta_{M} & \longrightarrow \\
\Delta^{2}(L) & \stackrel{\Delta^{2}(\varphi)}{\longrightarrow} \Delta^{2}(M) \longrightarrow 0 & \longrightarrow \operatorname{Coker}\left(\Delta^{2}(\varphi)\right) \longrightarrow 0
\end{array}
$$

we see that $\operatorname{Coker}\left(\Delta^{2}(\varphi)\right) \cong \operatorname{Coker}\left(\delta_{M}\right) \in \operatorname{Ker} \Gamma$, because of Lemma 1.1(d). Hence $\operatorname{Coker}\left(\Delta^{2}(\varphi)\right) \cong \operatorname{Coker}\left(\delta_{M}\right)=0$, so that $\delta_{M}$ is surjective and $M / \operatorname{Rej}_{U}(M) \cong \Delta^{2}(M)$ is $U$-tl.l.c. because of (a).

Proposition 1.6. Let $U_{R}$ be a cotilting module and let $S=\operatorname{End}\left(U_{R}\right)$. Then $U_{R}$ is U-tl.l.c. if and only if $\Delta \Gamma(S / I)=0$ for every left ideal I of $S$.

Proof. The module $U_{R}$ is $\Delta$-reflexive because of Lemma 1.1(a). Therefore, by Theorem 1.4, $U_{R}$ is $U$-tl.l.c. if and only if for any exact sequence of the form $0 \longrightarrow I \stackrel{i}{\longrightarrow} \Delta\left(U_{R}\right) \cong{ }_{S} S \longrightarrow S / I \longrightarrow 0$ it happens that $\operatorname{Coker}(\Delta(i)) \in \operatorname{Ker} \Delta$. Finally, from the previous sequence we get the exact sequence $0 \longrightarrow \Delta(S / I) \longrightarrow \Delta(S) \stackrel{\Delta(i)}{\longrightarrow} \Delta(I) \longrightarrow \Gamma(S / I) \longrightarrow 0$, which shows that $\operatorname{Coker}(\Delta(i)) \cong \Gamma(S / I)$.

We switch now to the case of a cotilting bimodule.

Corollary 1.7. Let ${ }_{S} U_{R}$ be a cotilting bimodule and let ${ }_{S} S\left(R_{R}\right.$, respectively) be noetherian. Then $U_{R}\left({ }_{S} U\right.$, respectively) is $U$-tl.l.c.

Proof. By assumption, for any left ideal $I$ of $S$ the cyclic module $S / I$ is finitely presented, and so it belongs to the class $\mathcal{C}$, as proved in $[\mathbf{C p}$, Proposition $5 \mathrm{~d}$ )]. Moreover, from $[\mathbf{C p}$, Theorem 6 a)], we get $\Gamma(\mathcal{C}) \subseteq \operatorname{Ker} \Delta$, so that $\Delta \Gamma(S / I)=0$. We finish the proof applying Proposition 1.6.

We are now ready to answer the question posed in [Cp, Remark 11].

Theorem 1.8. Let ${ }_{S} U_{R}$ be a cotilting bimodule. The following conditions are equivalent for any module $M_{R} \in \operatorname{Ker} \Gamma$ :

(1) $M_{R}$ is U-tl.l.c.

(2) $M_{R}$ is $\Delta$-reflexive and for all ${ }_{S} L \leq \Delta(M)$ we have $\Delta \Gamma(\Delta(M) / L)=0$. 
(3) Any $S$-submodule of $\Delta(M)$ is $\Delta$-reflexive.

Proof. (1) $\Leftrightarrow(2)$. Since $\Gamma \Delta(M)=0$, for any embedding $i:{ }_{S} L \hookrightarrow \Delta(M)$ we get $\operatorname{Coker}(\Delta(i))=\Gamma(\Delta(M) / L)$. Now apply Theorem 1.4.

$(2) \Rightarrow(3)$. For any ${ }_{S} L \leq \Delta(M)$ we get the exact sequence $\Delta^{2}(M) \rightarrow$ $\Delta(L) \rightarrow \Gamma(\Delta(M) / L) \rightarrow 0$ where, by assumption, since $(1) \Leftrightarrow(2), \Delta^{2}(M)$ is $U$-tl.l.c. and $\Gamma(\Delta(M) / L) \in \operatorname{Ker} \Delta$. So Corollary 1.5(a) applies, giving $\Delta(L)$ reflexive. Since $L$ is clearly in $\operatorname{Ker} \Gamma$, from Lemma 1.1(e) we obtain that $L$ is $\Delta$-reflexive.

$(3) \Rightarrow(2)$. By assumption $\Delta(M)$ is $\Delta$-reflexive, and so $M$ is $\Delta$-reflexive too, because of Lemma 1.1(e). Next, for any ${ }_{S} L \leq \Delta(M)$ we get the canonical exact sequence $0 \rightarrow L \rightarrow \Delta(M) \rightarrow \Delta(M) / L \rightarrow 0$, with both $L$ and $\Delta(M)$ $\Delta$-reflexive. Then $\Delta \Gamma(\Delta(M) / L)=0$ because of [Cp, Lemma $4 \mathrm{~d})]$.

Corollary 1.9. Let ${ }_{S} U_{R}$ be a cotilting bimodule. The following conditions are equivalent:

(1) every $\Delta$-reflexive right $R$-module is $U$-tl.l.c.,

(2) the class of all the $\Delta$-reflexive left $S$-modules is closed under submodules.

Proof. Apply (1) $\Leftrightarrow(3)$ of Theorem 1.8.

We now have the following connection between cotilting bimodules and those bimodules ${ }_{S} U_{R}$ that induce Colby's generalized Morita dualities [Cb2] in the sense that the classes of $\Delta$-reflexive modules are closed under extensions and submodules, and contain ${ }_{S} S$ and $R_{R}$, respectively.

Corollary 1.10. Let ${ }_{S} U_{R}$ be a cotilting bimodule. Then ${ }_{S} U_{R}$ induces a generalized Morita duality if and only if the class of the $\Delta$-reflexive modules coincides with the class of the $U$-torsionless linearly compact modules, both in $S$-Mod and in Mod- $R$.

Proof. For any cotilting bimodule ${ }_{S} U_{R}$, the regular modules ${ }_{S} S$ and $R_{R}$ are $\Delta$-reflexive, because of Lemma 1.1(a), and, similarly, any extension of two $\Delta$-reflexive modules is $\Delta$-reflexive too, because of $[\mathbf{C p}$, Proposition 5 a)]. Now apply Corollary 1.9.

\section{Morita duals of tilting bimodules.}

Originally cotilting bimodules arose as $k$-duals of tilting bimodules. Namely, consider two finite dimensional $k$-algebras $R$ and $S$, and denote by $D(-)$ the vector space $k$-duality. In this context a cotilting bimodule is just the dual $D\left({ }_{R} V_{S}\right)$ of a finite dimensional tilting bimodule ${ }_{R} V_{S}$, so cotilting theory for finite dimensional algebras is just a perfect dual of tilting theory. Moreover, since $D\left(R_{R}\right)$ is an injective cogenerator in $R$-Mod and adjunction induces a natural isomorphism of left $S$-modules $D\left(V_{S}\right) \cong \operatorname{Hom}_{R}\left({ }_{R} V_{S}, D\left(R_{R}\right)\right)$, it 
follows that $D\left(V_{S}\right)$ is a cotilting left $S$-module in our sense (see the proof of 2.4 below). Arguing in the same way for $D\left({ }_{R} V\right)$, we obtain that ${ }_{S} U_{R}=$ $D\left({ }_{R} V_{S}\right)$ is a cotilting bimodule in our sense.

Nevertheless, if we do not restrict our attention to finitely generated modules, cotilting theory is as far from tilting theory as Morita duality is from Morita equivalence. Even in this classical case, the theory seems to be quite hidden: We do not know, for instance, if the equivalent conditions of Corollary 1.9 hold true.

Obviously, a natural way to generalize this construction is to consider Morita duals of tilting bimodules. In this pursuit we are fortunate that standard methods yield the following extensions of the adjointness of the functors $\operatorname{Hom}_{A}(V,-)$ and $V \otimes_{S}-$ and of the contravariant functors $\operatorname{Hom}_{A}(-, W)$ and $\operatorname{Hom}_{R}(-, W)$ induced by bimodules ${ }_{A} V_{S}$ and ${ }_{A} W_{R}$ (see $\left.[\mathbf{A F}, \S 20]\right)$ :

Lemma 2.1. Let ${ }_{S} N$ and $M_{R}$ be modules and ${ }_{A} V_{S}$ and ${ }_{A} W_{R}$ be bimodules.

(a) If ${ }_{A} W$ is injective, then there are natural isomorphisms

$$
\operatorname{Hom}_{A}\left(\operatorname{Tor}_{n}^{S}(V, N), W\right) \cong \operatorname{Ext}_{S}^{n}\left(N, \operatorname{Hom}_{A}(V, W)\right)
$$

for $n=1,2, \ldots$.

(b) If ${ }_{A} W$ and $W_{R}$ are both injective, then there are natural isomorphisms $\operatorname{Ext}_{A}^{n}\left(V, \operatorname{Hom}_{R}(M, W)\right) \cong \operatorname{Ext}_{R}^{n}\left(M, \operatorname{Hom}_{A}(V, W)\right)$

for $n=1,2, \ldots$.

Proof. (a) This is [CE, page 120, Proposition 5.1].

(b) Being unable to find a reference for this part, we shall sketch a proof. Let

$$
\cdots \rightarrow P_{2} \rightarrow P_{1} \rightarrow P_{0} \rightarrow{ }_{A} V \rightarrow 0
$$

be a projective resolution of ${ }_{A} V$, and note that the conditions on $W$ yield an injective resolution

$$
0 \rightarrow \operatorname{Hom}_{A}(V, W) \rightarrow \operatorname{Hom}_{A}\left(P_{0}, W\right) \rightarrow \operatorname{Hom}_{A}\left(P_{1}, W\right)
$$$$
\rightarrow \operatorname{Hom}_{A}\left(P_{2}, W\right) \rightarrow \cdots
$$

of $\operatorname{Hom}_{A}(V, W)_{R}$. Then (see [R, Chapter 7]) one obtains the desired isomorphisms from the commutative diagram

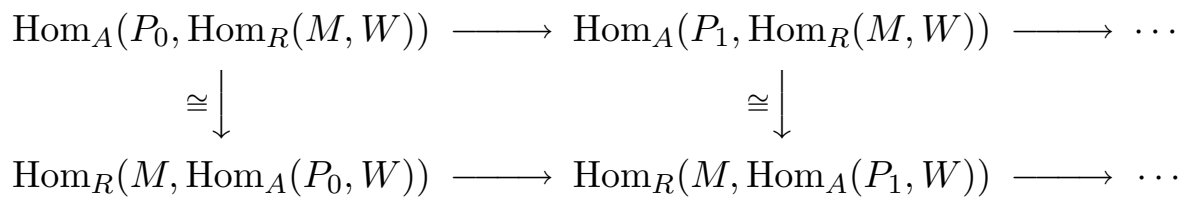

For the remainder of this section $A$ and $R$ are supposed to be Morita dual rings via faithfully balanced bimodule ${ }_{A} W_{R}$ that is a (linearly compact) injective cogenerator on both sides. Moreover we assume that ${ }_{A} V$ is 
a (linearly compact) tilting module with endomorphism $\operatorname{ring} S=\operatorname{End}\left({ }_{A} V\right)$, and we let

$$
{ }_{S} U_{R}=\operatorname{Hom}_{A}(V, W) .
$$

We futher assume that ${ }_{A} V$ is not projective (equivalently, not a (pro)generator), so that the bimodule ${ }_{S} U_{R}$ is not just another Morita bimodule.

For convenience sake, given any bimodule ${ }_{A} M_{B}$ we shall denote by $\Delta_{M}$ the two contravariant functors $\operatorname{Hom}_{?}\left(-,{ }_{A} M_{B}\right)$ and by $\Gamma_{M}$ their first derived functors $\operatorname{Ext}_{?}^{1}\left(-,{ }_{A} M_{B}\right)$, where $?=A$ or $B$. Also we put $H_{M}=$ $\operatorname{Hom}_{A}(M,-), T_{M}=M \otimes_{B}-, H_{M}^{\prime}=\operatorname{Ext}_{A}^{1}(M,-)$ and $T_{M}^{\prime}=\operatorname{Tor}_{1}^{B}(M,-)$.

Thus by adjointness we have

$$
\Delta_{U} \cong H_{V} \Delta_{W}: \text { Mod- } R \rightarrow S \text {-Mod }
$$

and

$$
\Delta_{U} \cong \Delta_{W} T_{V}: S \text {-Mod } \rightarrow \operatorname{Mod}-R
$$

and by Lemma 2.1

$$
\Gamma_{U} \cong H_{V}^{\prime} \Delta_{W}: \operatorname{Mod}-R \rightarrow S \text {-Mod }
$$

and

$$
\Gamma_{U} \cong \Delta_{W} T_{V}^{\prime}: S \text {-Mod } \rightarrow \text { Mod- } R .
$$

Also there are natural transformations

$$
\delta: \operatorname{id}_{\text {Mod- } R} \rightarrow \Delta_{U} \Delta_{U} \quad \text { and } \quad \delta: \operatorname{id}_{S-\operatorname{Mod}} \rightarrow \Delta_{U} \Delta_{U}
$$

and

$$
\gamma: \Gamma_{U} \Gamma_{U} \rightarrow \operatorname{id}_{\text {Mod- } R} \quad \text { and } \quad \gamma: \Gamma_{U} \Gamma_{U} \rightarrow \operatorname{id}_{S \text {-Mod }}
$$

with the $\delta$ 's via the usual evaluation maps, and the $\gamma$ 's derived from the natural transformations of the Tilting Theorem $[\mathbf{C b F u}, 1.4]$ and the $\Delta_{W}$ 's. Thus we obtain

Duality 2.2. There are dualities

$$
\begin{aligned}
\Delta_{U}: \mathcal{Y}_{R} \rightleftarrows{ }_{S} \mathcal{Y}: \Delta_{U} \\
\Gamma_{U}: \mathcal{X}_{R} \rightleftarrows{ }_{S} \mathcal{X}: \Gamma_{U}
\end{aligned}
$$

where the $\mathcal{Y}$ 's and $\mathcal{X}$ 's are the full subcategories on whose objects the $\delta$ 's and the $\gamma$ 's, respectively, are isomorphisms.

Let us denote by ${ }_{A} \mathcal{C}$ and $\mathcal{C}_{R}$ the classes of all linearly compact left $A$ and right $R$-modules, respectively. Moreover, $\left({ }_{A} \mathcal{T},{ }_{A} \mathcal{F}\right)$ denotes the torsion theory generated by the tilting module ${ }_{A} V$, and $\left({ }_{S} \mathcal{T},{ }_{S} \mathcal{F}\right)$ the torsion theory cogenerated by the cotilting module ${ }_{S} U=\operatorname{Hom}_{A}\left({ }_{A} V_{S},{ }_{A} W\right.$ ) (see the proof of 2.4 below).

By assumption, the bimodule ${ }_{A} W_{R}$ induces a duality of the form

$$
\Delta_{W}: \mathcal{C}_{R} \rightleftarrows{ }_{A} \mathcal{C}: \Delta_{W}
$$


and the tilting bimodule ${ }_{A} V_{S}$ induces the two equivalences

$$
H_{V}:{ }_{A} \mathcal{T} \rightleftarrows{ }_{S} \mathcal{F}: T_{V} \text { and } H_{V}^{\prime}:{ }_{A} \mathcal{F} \rightleftarrows{ }_{S} \mathcal{T}: T_{V}^{\prime}
$$

Therefore, letting

$$
{ }_{A} \mathcal{X}={ }_{A} \mathcal{C} \cap{ }_{A} \mathcal{T} \text { and }{ }_{A} \mathcal{Y}={ }_{A} \mathcal{C} \cap{ }_{A} \mathcal{F}
$$

we see that

$$
\begin{array}{ll}
\mathcal{X}_{R} \supseteq \Delta_{W}\left({ }_{A} \mathcal{Y}\right), & \mathcal{Y}_{R} \supseteq \Delta_{W}\left({ }_{A} \mathcal{X}\right), \\
{ }_{S} \mathcal{X} \supseteq H_{V}^{\prime}\left({ }_{A} \mathcal{Y}\right), & S \mathcal{Y} \supseteq H_{V}\left({ }_{A} \mathcal{X}\right) .
\end{array}
$$

Since ${ }_{A} V$ is a tilting module, ${ }_{A} V$ and ${ }_{A} W$ belong to ${ }_{A} \mathcal{X}$. Thus

$$
\begin{aligned}
& U_{R}=\Delta_{W}\left({ }_{A} V\right) \in \mathcal{Y}_{R}, \quad R_{R}=\Delta_{W}\left({ }_{A} W\right) \in \mathcal{Y}_{R}, \\
& { }_{S} U=H_{V}\left({ }_{A} W\right) \in{ }_{S} \mathcal{Y}, \quad{ }_{S} S=H_{V}\left({ }_{A} V\right) \in{ }_{S} \mathcal{Y},
\end{aligned}
$$

so, in particular, we have:

2.3. Balance. The bimodule ${ }_{S} U_{R}$ is faithfully balanced.

Since ${ }_{A} V$ is a *-module, ${ }_{S} U=\operatorname{Hom}_{A}\left({ }_{A} V_{S},{ }_{A} W\right)$ and ${ }_{A} W$ is an injective cogenerator, as in [CpToTr, 2.3 3)], we obtain:

2.4. Properties of ${ }_{S} U \cdot{ }_{S} U$ is a cotilting module.

One would hope that $U_{R}$ is one too. Perhaps not in general, but we do have the following:

\subsection{Properties of $U_{R}$.}

(a) There is an exact sequence $0 \rightarrow U_{R} \rightarrow W^{\prime} \rightarrow W^{\prime \prime} \rightarrow 0$, where $W^{\prime}, W^{\prime \prime} \in \operatorname{add}\left(W_{R}\right)$. In particular $U_{R}$ is finitely cogenerated and $\operatorname{inj} \operatorname{dim}\left(U_{R}\right) \leq 1$.

(b) There is an exact sequence $0 \rightarrow U^{\prime} \rightarrow U^{\prime \prime} \rightarrow W_{R} \rightarrow 0$, where $U^{\prime}, U^{\prime \prime} \in$ $\operatorname{add}\left(U_{R}\right)$. In particular $\operatorname{Ker} \Delta_{U} \cap \operatorname{Ker} \Gamma_{U}=0$.

(c) $\Delta_{W}\left({ }_{A} \mathcal{X}\right) \subseteq \operatorname{Ker} \Gamma_{U}$. In particular $\operatorname{Ext}_{R}^{1}(M, U)=0$ for all $M_{R} \hookrightarrow U_{R}^{n}$ ( $n$ finite).

Proof. (a) Since ${ }_{A} V$ is a tilting module, there is an exact sequence of the form $0 \rightarrow A^{\prime} \rightarrow A^{\prime \prime} \rightarrow{ }_{A} V \rightarrow 0$, with $A^{\prime}, A^{\prime \prime} \in \operatorname{add}\left({ }_{A} A\right)$. Now apply $\Delta_{W}$.

(b) Similarly to the previous case, applying $\Delta_{W}$ to the exact sequence $0 \rightarrow{ }_{A} A \rightarrow V^{\prime} \rightarrow V^{\prime \prime} \rightarrow 0$, where $V^{\prime}, V^{\prime \prime} \in \operatorname{add}\left({ }_{A} V\right)$, we obtain the required exact sequence. Finally, applying $\operatorname{Hom}_{R}(M,-)$ to that, we see that $\operatorname{Hom}_{R}(M, U)=0=\operatorname{Ext}_{R}^{1}(M, U)$ implies $\operatorname{Hom}_{R}(M, W)=0$, and so $M=0$.

(c) For any $M \in \Delta_{W}\left({ }_{A} \mathcal{X}\right)$ we clearly have $\Delta_{W}(M) \in{ }_{A} \mathcal{X} \subseteq{ }_{A} \mathcal{T}=$ $\operatorname{Ker} \operatorname{Ext}_{A}^{1}(V,-)$. Therefore, we see by Lemma 2.1(b) that $\operatorname{Ext}_{R}^{1}(M, U) \cong$ $\operatorname{Ext}_{A}^{1}\left(V, \Delta_{W}(M)\right)=0$.

From 2.3, 2.4 and 2.5 we immediately have:

Proposition 2.6. The bimodule ${ }_{S} U_{R}$ is a cotilting bimodule if and only if $\operatorname{Ext}_{R}^{1}\left(U^{\alpha}, U\right)=0$ for any cardinal $\alpha$. 


\section{Cotilting bimodules over noetherian serial rings.}

In $[\mathbf{C b F u}]$ Colby and Fuller determined all the tilting bimodules ${ }_{R} V_{S}$ over a noetherian serial ring $R$. In this concluding section we shall see that if $R$ has self-duality induced by ${ }_{R} W_{R}$ then ${ }_{S} U_{R}=\operatorname{Hom}_{R}(V, W)$ is a cotilting bimodule. Thus we obtain a large collection of cotilting bimodules (that are not even finitely generated) in addition to the classical ones over finite dimensional algebras.

According to [Wa, Theorem 5.11], a noetherian serial ring is a finite direct sum of indecomposable artinian serial rings and prime noetherian serial rings. Warfield proved that every finitely generated module and every injective module over such a ring is a direct sum of uniserial modules. The structure of artinian serial rings is well known (see $[\mathbf{A F}, \S 32]$ ).

Let $R$ be a prime noetherian serial ring with right Kupisch series

$$
e_{1} R, \ldots, e_{n} R
$$

so that, setting $J=\mathrm{J}(R)$

$$
e_{1} J \cong e_{2} R, \ldots, e_{n-1} J \cong e_{n} R \text { and } e_{n} J \cong e_{1} R
$$

(see $[\mathbf{C b F u}, \S 3])$. According to Warfield [Wa]

$$
e_{i} R>e_{i} J>e_{i} J^{2}>\ldots \quad \text { and } \quad R e_{i}>J e_{i}>J^{2} e_{i}>\ldots
$$

are complete lists of the submodules of $e_{i} R$ and $R e_{i}$, for $i=1, \ldots, n$. Thus, setting $S_{i}=e_{i} R / e_{i} J$, the composition factors of $e_{i} R$ are, from the top down,

$$
S_{i}, S_{i+1}, \ldots, S_{n}, S_{1}, S_{2}, \ldots, S_{n}, \ldots \text {. }
$$

On the other hand, as Warfield showed, every finitely generated indecomposable $R$-module is uniserial. It follows that the indecomposable injective $R$-modules are also uniserial. There are just $n+1$ indecomposable injective right $R$-modules

$$
E_{1}=\mathrm{E}\left(S_{1}\right), \ldots, E_{n}=\mathrm{E}\left(S_{n}\right) \text { and } E_{0}
$$

with $\operatorname{Soc}\left(E_{0}\right)=0$, each $E_{i}$ is artinian, and for any $i=1, \ldots, n$ the submodules of $E_{i}$ are

$$
0<\operatorname{Soc}\left(E_{i}\right)<\operatorname{Soc}^{2}\left(E_{i}\right)<\ldots
$$

where $\operatorname{Soc}^{k}(M)=\operatorname{Ann}_{M}\left(J^{k}\right)$. And the composition factors of $E_{i}$, from the bottom up, are

$$
S_{i}, S_{i-1}, \ldots, S_{1}, S_{n}, S_{n-1}, \ldots, S_{1}, \ldots
$$

while the composition factor of $E_{0}$ are

$$
\ldots S_{n}, \ldots, S_{i}, S_{i-1}, \ldots S_{1}, S_{n}, S_{n-1}, \ldots, S_{i}, S_{i-1}, \ldots S_{1}, \ldots .
$$

In particular any proper factor of an indecomposable injective module is the injective envelope of its socle, and every proper submodule of $E_{0}$ is isomorphic to an indecomposable projective module. 
Lemma 3.1. Let $R$ be a noetherian serial ring. If $X_{R}$ is an indecomposable $R$-module of finite length, then for any cardinal $\alpha$ there is a cardinal $\gamma$ such that $X^{\alpha} \cong X^{(\gamma)}$.

Proof. Let $Q=R / \operatorname{Ann}_{R}(X)$. Then $X_{Q}$ is a faithful indecomposable module over the artinian QF-3 ring $Q$. Thus $X$ is the unique indecomposable injective projective right $Q$-module (see $[\mathbf{A F}, \S 31$ and $\S 32]$ ). But $X^{\alpha}$ is both injective and, since $Q$ is artinian, projective. Moreover $X_{Q}^{\alpha}$ is a direct sum of indecomposable modules, since $Q$ is artinian.

Lemma 3.2. Let $R$ be a prime noetherian serial ring with indecomposable injective modules $E_{1}, \ldots, E_{n}$ and $E_{0}$ as above. Then for any cardinal $\alpha$ there are cardinals $\beta, \gamma$ such that $E_{i}^{\alpha} \cong E_{i}^{(\beta)} \oplus E_{0}^{(\gamma)}$.

Proof. Since $R$ is semiperfect and $J$ is finitely generated, we see that

$$
\operatorname{Soc}\left(E_{i}^{\alpha}\right)=\operatorname{Ann}_{E_{i}^{\alpha}}(J)=\operatorname{Ann}_{E_{i}}(J)^{\alpha}=\operatorname{Soc}\left(E_{i}\right)^{\alpha} .
$$

But if $i \neq j$ then $\operatorname{Soc}\left(E_{i}\right) e_{j}=0$. Thus $\operatorname{Soc}\left(E_{i}^{\alpha}\right)=S_{i}^{(\beta)}$. So we see that $E_{i}^{\alpha} \cong E_{i}^{(\beta)} \oplus E$, with $\operatorname{Soc}(E)=0$. But the only indecomposable injective with zero socle is $E_{0}$, so $E \cong E_{0}^{(\gamma)}$.

Proposition 3.3. If $U$ is a finitely cogenerated module over a noetherian serial ring $R$ such that $\operatorname{Ext}_{R}^{1}(U, U)=0$, then $\operatorname{Ext}_{R}^{1}\left(U^{\alpha}, U\right)=0$ for any cardinal $\alpha$.

Proof. Since $U$ is finitely cogenerated, we have

$$
U=E_{i_{1}} \oplus \cdots \oplus E_{i_{k}} \oplus X_{1} \oplus \cdots \oplus X_{l}
$$

where $E_{i_{j}}=\mathrm{E}\left(S_{i_{j}}\right), j=1, \ldots, k$, and $X_{i}, i=1, \ldots, l$, are uniserial modules of finite length. Thus by Lemmas 3.1 and 3.2 we have

$$
U^{\alpha}=E_{i_{1}}^{\left(\beta_{1}\right)} \oplus \cdots \oplus E_{i_{k}}^{\left(\beta_{k}\right)} \oplus E_{0}^{(\gamma)} \oplus X_{1}^{\left(\delta_{1}\right)} \oplus \cdots \oplus X_{l}^{\left(\delta_{l}\right)} .
$$

Now, since $\operatorname{Ext}_{R}^{1}\left(-, X_{i}\right)$ converts direct sums to direct products, we need only check that $\operatorname{Ext}_{R}^{1}\left(E_{0}, X_{i}\right)=0$ for all $i=1, \ldots, l$. To this end, consider the minimal injective resolution

$$
0 \longrightarrow X_{i} \longrightarrow E_{i} \stackrel{\nu}{\longrightarrow} E_{j} \longrightarrow 0 .
$$

Here we need to show that

$$
\operatorname{Hom}_{R}\left(E_{0}, E_{i}\right) \stackrel{\operatorname{Hom}_{R}\left(E_{0}, \nu\right)}{\longrightarrow} \operatorname{Hom}_{R}\left(E_{0}, E_{j}\right) \longrightarrow 0
$$

is exact. So let $0 \neq \beta \in \operatorname{Hom}_{R}\left(E_{0}, E_{j}\right)$ with $K=\operatorname{Ker}(\beta)$. Then there is $m \in \mathbb{N}$ such that $E_{0} / K J^{m} \cong E_{i_{1}}$. But $\operatorname{Ext}_{R}^{1}\left(E_{i_{1}}, X_{i}\right)=0$, being a direct summand of $\operatorname{Ext}_{R}^{1}(U, U)$, so

$$
\operatorname{Hom}_{R}\left(E_{0} / K J^{m}, E_{i}\right) \stackrel{\operatorname{Hom}_{R}\left(E_{0} / K J^{m}, \nu\right)}{\longrightarrow} \operatorname{Hom}_{R}\left(E_{0} / K J^{m}, E_{j}\right) \longrightarrow 0
$$


is exact. Thus, setting $\eta: E_{0} \rightarrow E_{0} / K J^{m}$, we have a commutative diagram

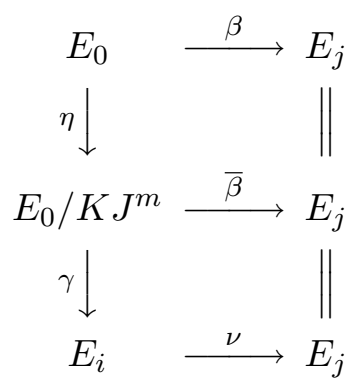

which shows that $\operatorname{Hom}_{R}\left(E_{0}, \nu\right)(\gamma \circ \eta)=\nu \circ \gamma \circ \eta=\bar{\beta} \circ \eta=\beta$.

Theorem 3.4. Let $R$ be a noetherian serial ring with self-duality induced by a bimodule ${ }_{R} W_{R}$. If ${ }_{R} V$ is a tilting module and $S=\operatorname{End}\left({ }_{R} V\right)$, then ${ }_{S} U_{R}=\operatorname{Hom}_{R}(V, W)$ is a cotilting bimodule.

Proof. According to Proposition 2.6, it only remains to observe that $\operatorname{Ext}_{R}^{1}\left(U^{\alpha}, U\right)=0$ for any $\alpha$. And this is true thanks to Proposition 3.3, since $\operatorname{Ext}_{R}^{1}(U, U)=0$ and $U_{R}$ is finitely cogenerated because of 2.5(c) and (a).

Let us pause to point out a couple of facts about self-duality for noetherian serial rings.

Proposition 3.5. If $R$ is a left linearly compact indecomposable prime noetherian serial ring, then $R$ has a self-duality.

Proof. Assume, as we may, that $R$ is basic. Let $E=E_{1} \oplus \cdots \oplus E_{n}$ be the minimal cogenerator. Then $E$ is artinian, hence linearly compact. Thus, setting $S=\operatorname{End}\left({ }_{R} E\right), S_{S}$ is linearly compact and the bimodule ${ }_{R} E_{S}$ defines a Morita duality. Now it is easy to see that $A_{k}=\operatorname{Ann}_{E}\left(J^{k}\right)$ is the minimal cogenerator over $R / J^{k}$, and that the bimodule ${ }_{R / J^{k}} A_{k S / \operatorname{Ann}_{S}\left(A_{k}\right)}$ defines a Morita duality. But $R / J^{k}$ is a basic QF-ring (see $[\mathbf{A F}, \S 32.6]$ ) and hence $R / J^{k} A_{k} \cong{ }_{R / J^{k}} R / J^{k}$. But then

$$
S / \operatorname{Ann}_{S}\left(A_{k}\right) \cong \operatorname{End}\left(R / J^{k} A_{k}\right) \cong R / J^{k}
$$

as rings. Now both $\left\{J^{k} \mid k \geq 1\right\}$ and $\left\{\operatorname{Ann}_{S}\left(A_{k}\right) \mid k \geq 1\right\}$ are downward directed sets of ideals with $\cap_{k} J^{k}=0$ [Wa, Theorem 5.11] and so $\cap_{k} \operatorname{Ann}_{S}\left(A_{k}\right)=0$. Therefore, since ${ }_{R} R$ and $S_{S}$ are both linearly compact, we have

$$
R \cong \lim _{\longleftarrow} R / J^{k} \cong \lim _{\longleftarrow} S / \operatorname{Ann}_{S}\left(A_{k}\right) \cong S \text {. }
$$

As Warfield [Wa] showed, a prime noetherian serial $\operatorname{ring} R$ is isomorphic to the $n \times n(D: M)$-upper triangular matrix ring $\operatorname{UTM}_{n}(D: M)$, consisting of those matrices over a local noetherian serial ring $D$ whose entries below 
the main diagonal all come from the unique maximal ideal $M$ of $D$. It follows from Proposition 3.5 and [X, Theorem 4.3, Lemma 4.9 and Proposition 3.3] that $R$ has self-duality if and only if $D$ is linearly compact. According to $[\mathbf{W b}]$ and $[\mathbf{D i M l}]$, any artinian serial ring has self-duality. Thus from Proposition 3.5 and $[\mathbf{M u}]$ (see again $[\mathbf{X}$, Theorem 4.3]) we have:

Proposition 3.6. A noetherian serial ring has a self-duality if and only if it is left (equivalently right) linearly compact.

Finally, we note that any tilting module ${ }_{R} V$ over a hereditary noetherian ring (which was shown to be a finitistic cotilting module in $[\mathbf{C b F u}]$ ) satisfies at least two of the three conditions needed to be a cotilting module in our sense whenever $R$ has selfduality.

Proposition 3.7. Let $R$ be a hereditary linearly compact noetherian serial ring and let ${ }_{R} V$ be a tilting module. Then ${ }_{R} V$ is a finitistic cotilting module with $\operatorname{Ext}_{R}^{1}\left(V^{\alpha}, V\right)=0$ for all cardinal numbers $\alpha$.

Proof. According to $\left[\mathbf{C b F u}\right.$, Proposition 2.1], ${ }_{R} V$ is a finitistic cotilting module, and since it is finitely generated

$$
{ }_{R} V=P \oplus T,
$$

with $P$ finitely generated projective and $T=T_{1} \oplus \cdots \oplus T_{l}$, with all the $T_{i}$ 's uniserial modules of finite length. Since $\operatorname{Ext}_{R}^{1}(V, V)=0$, and since, by Lemma 3.1,

$$
V^{\alpha}=P^{\alpha} \oplus T_{1}^{\left(\delta_{1}\right)} \oplus \cdots \oplus T_{l}^{\left(\delta_{l}\right)},
$$

it only remains to show that $\operatorname{Ext}_{R}^{1}\left(P^{\alpha}, P\right)=0$ and $\operatorname{Ext}_{R}^{1}\left(P^{\alpha}, T_{i}\right)=0$ for $i=1, \ldots, l$.

Let ${ }_{R} W_{R}$ induce a self-duality and observe that the canonical right $R$ isomorphism

$$
R \longrightarrow \operatorname{Hom}_{R}\left(\operatorname{Hom}_{R}\left(R_{R}, W\right), W\right) \longrightarrow \operatorname{Hom}_{R}\left({ }_{R} W_{R},{ }_{R} W_{R}\right)
$$

is also a left $R$-map. Now $P^{\alpha}$ is flat by Chase's Theorem [AF, 19.20], since $R$ is noetherian, and so by Lemma 2.1 (a)

$$
\begin{aligned}
\operatorname{Ext}_{R}^{1}\left(P^{\alpha},{ }_{R} R\right) & \cong \operatorname{Ext}_{R}^{1}\left(P^{\alpha}, \operatorname{Hom}_{R}\left({ }_{R} W_{R},{ }_{R} W\right)\right) \\
& \cong \operatorname{Hom}_{R}\left(\operatorname{Tor}_{1}^{R}\left(W, P^{\alpha}\right), W\right)=0 .
\end{aligned}
$$

Thus, assuming, as we may, that $P$ is a direct summand of ${ }_{R} R$, we do have

$$
\operatorname{Ext}_{R}^{1}\left(P^{\alpha}, P\right)=0 \text {. }
$$

On the other hand, if $T_{i}$ has length $m$, and $A=R / J^{m}$, then ${ }_{A} T_{i}$ is injective $[\mathbf{A F}$, Theorem 32.6] and

$$
{ }_{R} T_{i} \cong \operatorname{Hom}_{A}\left({ }_{A} A_{R},{ }_{A} T_{i}\right),
$$


so that

$$
\begin{aligned}
\operatorname{Ext}_{R}^{1}\left(P^{\alpha},{ }_{R} T_{i}\right) & \cong \operatorname{Ext}_{R}^{1}\left(P^{\alpha}, \operatorname{Hom}_{A}\left({ }_{A} A_{R},{ }_{A} T_{i}\right)\right) \\
& \cong \operatorname{Hom}_{R}\left(\operatorname{Tor}_{1}^{R}\left(A_{R}, P^{\alpha}\right),{ }_{A} T_{i}\right)=0 .
\end{aligned}
$$

Remark 3.8. (1) Krause and Saorín [KrSa, Proposition 3.8] have recently shown that if $M_{R}$ is a finitely generated module, then every $M^{\alpha}$ is isomorphic to a direct summand of some $M^{(\delta)}$ if and only if $S=\operatorname{End}\left(M_{R}\right)$ is left coherent and right perfect and ${ }_{S} M$ is finitely presented. Thus we see that if $R$ is right artinian and (hence) $S$ is left artinian in a cotilting triple $(S, U, R)$ in the sense of $[\mathbf{C b} 1, \S 2]$, then $\operatorname{Ext}_{R}^{1}\left(U^{\alpha}, U\right)=0=\operatorname{Ext}_{S}^{1}\left(U^{\alpha}, U\right)$ for any cardinal $\alpha$.

(2) Over rings of finite representation type, cotilting triples yield more examples of cotilting modules. Indeed, in a cotiling triple $(S, U, R)$, if it happens that $R$ is a ring of finite representation type (so that every $R$-module is a direct sum of finitely generated modules), then since $U_{R}$ is a finitistic cotilting module [Cb1, Theorem 3.3], we also have $\operatorname{Ker} \operatorname{Hom}_{R}\left(-, U_{R}\right) \cap$ $\operatorname{Ker} \operatorname{Ext}_{R}^{1}\left(-, U_{R}\right)=0$, so that $U_{R}$ is a cotilting module in the present sense. If in addition $S$ has finite representation type (in particular, if $R$ is hereditary [ $\mathbf{C b F u}$, Proposition 2.2]), then ${ }_{S} U_{R}$ is a cotilting bimodule.

Acknowledgments. This paper was written while R. Colpi was visiting the University of Iowa in April-May '98, and he wishes to express his gratitude to this University and especially to Kent Fuller for the great hospitality.

\section{References}

[AF] F.D. Anderson and K.R. Fuller, Rings and Categories of Modules (2nd edition), Springer, New York, 1992.

$[\mathrm{BrBu}] \quad \mathrm{S}$. Brenner and M. Butler, Generalizations of the Bernstein-GelfandPonomarev reflection functors, in 'Proc. ICRA II (Ottawa, 1979)', LNM 832, Springer, Berlin, (1980), 103-169.

[CE] H. Cartan and S. Eilenberg, Homological Algebra, Princeton University Press, 1956.

[Cb1] R.R. Colby, A generalization of Morita duality and the tilting theorem, Comm. Algebra, 17(7) (1989), 1709-1722.

[Cb2] _ A cotilting theorem for rings, in 'Methods in Module Theory', M. Dekker, New York, (1993), 33-37.

[CbFu] R.R. Colby and K.R. Fuller, Tilting, cotilting and serially tilted rings, Comm. Algebra, 18(5) (1990), 1585-1615.

[Cp] R. Colpi, Cotilting bimodules and their dualities, to appear in '1998 Murcie Euroconference Proceedings', Marcel Dekker.

[CpDeTo] R. Colpi, G. D'Este and A. Tonolo, Quasi-tilting modules and counter equivalences, J. Algebra, 191 (1997), 461-494. 
[CpToTr] R. Colpi, A. Tonolo and J. Trlifaj, Partial cotilting modules and the lattices induced by them, Comm. Algebra, 25 (1997), 3225-3237.

[DiMl] F. Dischinger and W. Müller, Einreihig zerlegbare Ringe sind selbstdual, Arch. Math., 43 (1984), 132-136.

[GpGaWi] J.L. Gómez Pardo, P.A. Guil Asensio and R. Wisbauer, Morita dualities induced by the $M$-dual functors, Comm. Algebra, 22 (1994), 5903-5934.

[H] D. Happel, Triangulated Categories in the Representation Theory of Finite Dimensional Algebras, Cambridge Univ. Press, Cambridge, 1988.

[HaRi] D. Happel and C.M. Ringel, Tilted algebras, Trans. Amer. Math. Soc., 274 (1982), 399-443.

[KrSa] H. Krause and M. Saorín, On minimal approximations of modules, Preprint, 1998.

[Mu $\quad$ B.J. Müller, Linear compactness and Morita duality, J. Algebra, 16 (1970), 60-66.

[R] J.J. Rotman, An Introduction to Homological Algebra, Academic Press, New York, 1979.

[S] B. Stenström, Rings of Quotients, Springer-Verlag, Berlin, Heidelberg, New York, 1975.

[Wa] R.B. Warfield, Serial rings and finitely presented modules, J. Algebra, 37 (1975), 187-222.

[Wb] J. Waschbüsch, Self-duality of serial rings, Comm. Algebra, 14 (1986), 581589 .

[X] W. Xue, Rings with Morita Duality, LNM 11523, Springer-Verlag, Berlin, Heidelberg, New York, 1992.

Received June 23, 1998 and revised October 20, 1998. Research partially supported by grant CNR-GNSAGA.

UNIVERSitÀ Di PADOVA

Via Belzoni 7, 35131 Padova

ITALY

E-mail address: colpi@math.unipd.it

UNIVERSITY OF IOWA

IowA City, IA 52242

E-mail address: kfuller@math.uiowa.edu 